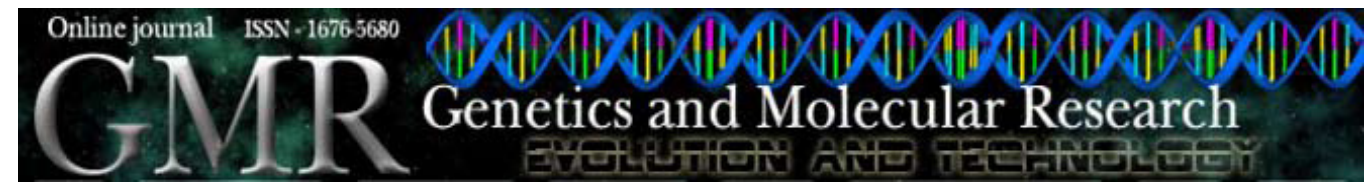

\title{
Mutational analysis of genes p14ARF, p15INK4b, p16INK4a, and PTEN in human nervous system tumors
}

\author{
L.O. Almeida ${ }^{1}$, A.C. Custódio ${ }^{1}$, J.J. Araújo ${ }^{1}$, J.A. Rey ${ }^{2}$, \\ J.R.W. Almeida ${ }^{2}$, M.J. Santos ${ }^{2}$, C.A. Clara ${ }^{2}$ and C. Casartelli ${ }^{1}$ \\ ${ }^{1}$ Laboratório de Oncogenética, Departamento de Genética, \\ Faculdade de Medicina de Ribeirão Preto, Universidade de São Paulo, \\ Ribeirão Preto, SP, Brasil \\ ${ }^{2}$ Unidad de Investigación, Laboratorio de Oncogenética Molecular, \\ Hospital Universitário La Paz, Madrid, Spain \\ ${ }^{3}$ Fundação Pio XII, Hospital de Câncer de Barretos, Barretos, SP, Brasil \\ Corresponding author: L.O. Almeida \\ E-mail: lu_olive@yahoo.com
}

Genet. Mol. Res. 7 (2): 451-459 (2008)

Received March 14, 2008

Accepted May 2, 2008

Published May 27, 2008

\begin{abstract}
The cancer is one of the most common and severe problems in clinical medicine, and nervous system tumors represent about $2 \%$ of the types of cancer. The central role of the nervous system in the maintenance of vital activities and the functional consequences of the loss of neurons can explain how severe brain cancers are. The cell cycle is a highly complex process, with a wide number of regulatory proteins involved, and such proteins can suffer alterations that transform normal cells into malignant ones. The INK4 family members (CDK inhibitors) are the cell cycle regulators that block the progression of the cycle through the $\mathrm{R}$ point, causing an arrest in G1 stage. The p14ARF (alternative reading frame) gene is a tumor suppressor that inhibits p53 degradation during the progression of the cell cycle. The PTEN gene is related to the induction of growth suppression through cell cycle arrest, to apoptosis and to the inhibition of cell adhesion and migration. The purpose of the present study was to assess the mutational state of the genes p14ARF, p15INK4b, p16INK4a, and PTEN in 64 human nervous system tumor samples. Homozygous deletions were found
\end{abstract}


in exon 2 of the p15INK $4 \mathrm{~b}$ gene and exon 3 of the p16INK4a gene in two schwannomas. Three samples showed a guanine deletion ( 63 codon) which led to a loss of heterozygosity in the p15 gene, and no alterations could be seen in the PTEN gene. Although the group of patients was heterogeneous, our results are in accordance with other different studies that indicate that homozygous deletion and loss of heterozygosity in the INK4 family members are frequently observed in nervous system tumors.

Key words: p14ARF; p15INK4b; p16INK4a; PTEN; Mutations

\section{INTRODUCTION}

Although the incidence of human nervous system tumors is small when compared to other neoplasms, they are among the most serious human malignancies because they affect the organ responsible for the coordination and integration of all life activities. Human nervous system tumors represent about $2 \%$ of all types of cancer, with an incidence estimated between 4.2 and 5.4 per 100,000 individuals per year (Ohgaki and Kleihues, 2005).

The formation of brain tumors is a complex process, which involves an accumulation of genetic alterations that together give each tumor its own molecular identification, making it possible to group them into distinct molecular types, and still distinguish them within the same group in relation to their biological behavior (Rocha, 2001). Pilocytic astrocytomas (grade I) frequently show allelic losses in 17p and 17q, including the TP53 and the NF1 genes. Around $60 \%$ of the diffuse astrocytomas (grade II) have deletions or mutations in TP53, allelic losses of RB1 and NF2 and overexpression of PDGFA. Glioblastomas have the highest number of alterations among the glial tumors; TP53 demonstrates mutations in 37\% of the cases of glioblastomas, and other genes such as p14ARF and HDM2, which control p53 levels in the cell, are also frequently mutated. The PTEN gene seems to be mutated in $45 \%$ of glioblastomas, and genes involved in the RB1 pathway of cell cycle control demonstrate abnormalities in $90 \%$ of the cases (Collins, 2004).

The cascade RB/E2F/INK4/cyclin D - Cdk4-6 - acts in an important way in the control of cell growth, integrating multiple mitogenic and anti-mitogenic stimuli. The INK4 family members act in a competitive way linking directly to the CDKs 4 and 6 and preventing the association with cyclin $\mathrm{D}$, keeping the RB proteins active and preventing the progression of the cell cycle through the R point, causing an arrest in G1 (Sherr and Roberts, 1999). The p14ARF activity is related to the p53 regulation in response to uncontrolled growth or an oncogenic stress. ARF binds to and inactivates the HDM2 protein that negatively regulates p53 (Ghimenti et al., 2003). In a small 25-kb region of the human genome (9p21), the locus encodes three important related tumor suppressor genes (p14ARF, p15INK4b and p16INK4a). The INK4/ARF locus is deleted in a wide variety of tumors, including melanoma, pancreatic adenocarcinoma, gliomas, leukemias, and bladder carcinomas (Cánepa et al., 2007). The p15INK4b gene has a different open reading frame, while p16INK4a and p14ARF genes have different first exons, and due to splicing, they share the same second and third exons. However, because of an alternative reading frame, their proteins are not isoforms and their amino acids do not show homology (Kim and Sharpless, 2006).

The PTEN gene was first described in 1997, and its inactivation takes part in the formation of several types of malignant tumors such as glioblastomas, breast and prostate tumors, endometrial neoplasias, and hematological malignancies (Rasheed et al., 1995; Li et al., 1997; Liu et al., 2005; Maehama, 2007). It is a tumor suppressor gene located on chromosome 10 ( $\mathrm{Li}$ and Sun, 
1997; Di Vizio et al., 2005), which induces the suppression of growth through the arrest of the cell cycle and/or apoptosis induction and inhibits cell adhesion and migration (Marino et al., 2002; Liu et al., 2005). The PTEN gene acts like an inhibitor factor of the phosphatidylinositol 3-kinase pathway, and its function is to transfer a phosphate group to phosphatidylinositol 4-5 biphosphate, giving rise to phosphatidylinositol 3,4,5-trisphosphate.

Phosphatidylinositol 3,4,5-trisphosphate along with other factors sends signals to activate the proto-oncogene $\mathrm{PKB} / \mathrm{AKT}$ pathway, which leads to cell proliferation and inhibition of apoptosis (Sansal and Sellers, 2004; Chu and Tarnawski, 2004; Maehama, 2007). The PTEN gene can also act on the focal adhesion kinase dephosphorylating the signal transduction molecules, potentially inhibiting cellular migration and adhesion. Therefore, the loss of the PTEN gene can lead to cell migration and a high tendency to metastasis (Gunther et al., 2003; Chu and Tarnawski, 2004; Maehama, 2007).

The focus of this study was to assess the mutational profile of the tumor suppressor genes of major importance to the genesis and to the progression of different types of human cancers, genes directly involved in the regulation of the cell cycle, such as p16INK4a and p15INK4b, and genes involved in the regulation of other proteins connected to cancer such as p14ARF and PTEN.

\section{MATERIAL AND METHODS}

Sixty-four samples of nervous system tumors, surgically removed from previously untreated patients under care at the Neurosurgery Department of Fundação Pio XII, Cancer Hospital of Barretos, Barretos, SP, Brazil, were analyzed.

The samples, classified according to the WHO criteria were: 15 meningiomas, 5 schwannomas, 15 gliomas (grades I and II), 17 gliomas (grades III and IV), 8 metastatic tumors, and 4 embryonic tumors.

For DNA extraction, approximately $1 \mathrm{~cm}^{3}$ of freshly microdissected samples was used. DNA extraction was performed using proteinase $\mathrm{K}$ and phenol-chloroform according to the method described by Rey et al. (1992). The primers used for polymerase chain reaction-single-strand conformation polymorphism (PCR-SSCP) analysis were constructed using the Gene Runner program (version 3.05, Hasting Software, Inc.) and are shown in Table 1.

\begin{tabular}{|c|c|c|}
\hline Exon & Primer sense & Primer antisense \\
\hline P14E1 & 5'-TGGGGGTGAAGGTGGGGGGC-3' & 5'-CGGTTATCTCCTCCTCCTCC-3' \\
\hline P15E1 & 5'-GGAGCAGCGTGGGAAAGAAGGGG-3' & 5'-GATCTAGGTTCCAGCCCCGA-3' \\
\hline P15E2 & 5'-AACACCAGACATCAGAGACC-3' & 5'-AATAAAGTCGTTGTGGGCGG-3' \\
\hline P16E1 & 5'-GAGAGGGGGAGAGCAGGCAG-3' & 5'-AGAGTCGCCCGCCATCCCCT-3' \\
\hline P16E2 & 5'-CTGCTTGGCGGTGAGGGGGC-3' & 5'-TCAGGCCGTCCCACCGATTG-3' \\
\hline P16E3 & 5'-ACGGCAAGAGAGGAGGGCGG-3' & 5'-AAAGCGGGGTGGGTTGTGGC-3' \\
\hline PTEN E1 & 5'-TCTGCCATCTCTCTCCTCCT-3' & 5'-CCGCAGAAATGGATACAGGT-3' \\
\hline PTEN E2 & 5'-TGACCACCTTTTATTACTCCA-3' & 5'-AGTATCTTTTTCTGTGGC-3' \\
\hline PTEN E3 & 5’-TGTTAATGGTGGCTTTTTG-3' & 5'-TGTTAATGGTGGCTTTTTG-3' \\
\hline PTEN E4 & 5'-CCTAAGTTCAAAAGATAAC-3' & 5'-TACAGTCTATCGGGTTTA-3' \\
\hline PTEN E5A & 5'-TTCTTATTCTGAGGTTATC-3' & 5'-GCACATATCATTACACCAG-3' \\
\hline PTEN E5B & 5'-GCACATATCATTACACCAG-3' & 5'-GAAGAGGAAAGGAAAAACATC-3' \\
\hline PTEN E6 & 5'-CTTCTCTTTTTTTTCTGTCCACC-3' & 5'-AGCACTTACTGCAAGTTCCGCCAC-3' \\
\hline PTEN E7 & 5’-ATCGTTTTTGACAGTTTG-3' & 5'-CTCCCAATGAAAGTAAAG-3' \\
\hline PTEN E8A & 5'-CAGATAACTCAGATTGCC-3' & 5'-TTTCTACTTTTTCTGAGG-3' \\
\hline PTEN 8B & 5'-CCAGGACCAGACCAAACC-3' & 5'-ACATCACATACATACAAGTC-3' \\
\hline PTEN 9 & 5'-GTCATATTTGTGGGTTTTC-3' & 5'-GGTGTTTTATCCCTCTTG-3' \\
\hline
\end{tabular}


PCR was carried out in a final volume of $25 \mu \mathrm{L}$ containing 50 ng DNA, $50 \mu \mathrm{M}$ dNTPs (Amersham Biosciences), 1X PCR buffer (Biotools, Madrid, Spain) with $2 \mathrm{mM} \mathrm{MgCl}, 0.4$ $\mu \mathrm{M}$ of each oligonucleotide (primer) (Invitrogen, USA), and 0.5 U DNA polymerase (Biotools). PCR was performed utilizing the programmable Mastercycler thermocycler (Eppendorf, Inc.), and the conditions for the amplification were: one initial denaturation step at $94^{\circ} \mathrm{C}$ for $5 \mathrm{~min}$, followed by 35 denaturation cycles of $30 \mathrm{~s}$ at $94^{\circ} \mathrm{C}, 30 \mathrm{~s}$ of annealing at $53-69^{\circ} \mathrm{C}$ (specific for each primer), and $30 \mathrm{~s}$ of extension at $72^{\circ} \mathrm{C}$, followed by a final elongation cycle at $72^{\circ} \mathrm{C}$ for $5 \mathrm{~min}$.

To screen the possible genetic alterations, the PCR-SSCP technique was applied (Kukita et al., 1997). For the subsequent sequencing, the PCR products were purified with ExoSAPIT kit (USB - Amersham Biosciences), and the DYEnamic ET Terminator Cycle Sequencing Kit (Amersham Biosciences) was used. As positive control, a polymorphic mutation (dbSNP: rs2156310) of exon 1 of the TFF gene was used. It consists of a transition of C/T in region 5'-UTR of the mRNA at TFF 1 at position 38, and the primers are (F): 5'-GCCTGCCCTATAAAATCC3'and (R): 5'-CACAGAGCAGGAAGAAGC-3', generating a 189-bp fragment that presents a characteristic migration pattern when submitted to the SSCP technique.

In the investigation of homozygous deletions, the samples were submitted to the comparative multiplex PCR technique (Ono et al., 1996). Exons of the PTEN gene previously amplified with the same samples and primers of the $\beta$-globin gene were used as positive controls. The product of the reaction was submitted to electrophoresis on $2 \%$ agarose gel, and the intensity of the DNA fragments was evaluated using the 1-D Image Analysis Software (Kodak Digital Science) and compared to the control samples.

After sequencing, some samples were submitted to enzymatic digestion, to confirm the possibility of deletion of a nucleotide. AluI enzyme (Invitrogen) was used, whose restriction site is 5'-AG/CT-3' and 3'-TC/GA-5'. The PCR product was precipitated with absolute ethanol, and $46 \mu \mathrm{L}$ water, $5 \mu \mathrm{L}$ buffer (React1) and $1 \mu \mathrm{L}$ AluI were added. The samples were incubated at $37^{\circ} \mathrm{C}$ overnight and were analyzed on $2 \%$ polyacrylamide gel.

\section{RESULTS}

No alterations were found in the exons of the genes PTEN, p14ARF, p15INK4b exon 1 or gene p16INK4a exons 1 and 2. Homozygous deletions were observed in all exon 2 of the p15INK $4 b$ gene and in exon 3 of the p16 INK4a gene in two samples of schwannomas (Figure 1A and B).
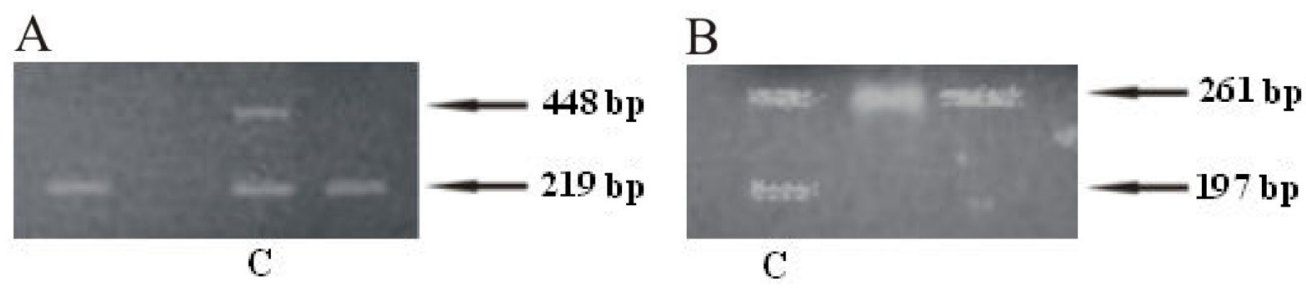

Figure 1. Electrophoresis on agarose gel. A. Homozygous deletion in the p15INK4b gene, C (control) shows bands of 448 and $219 \mathrm{bp}$ of exon 2 of p15INK4b and exon 8B of the PTEN gene, respectively. B. Homozygous deletion in the p16INK4a gene, C (control) shows bands of 261 and $197 \mathrm{bp}$ regarding exon 8A of PTEN and exon 3 of p16INK4a, respectively. 
After the sequencing reaction, a deletion of a $\mathrm{G}$ base (guanine) was found in exon 2, codon $63(\mathrm{Glu})$ of the $\mathrm{p} 15$ protein, only in the sense strand in three samples (astrocytoma grade II, glioblastoma and metastasis) (Figure 2A to F). The deletion was confirmed with enzymatic digestion with the AluI enzyme (Figure 3).
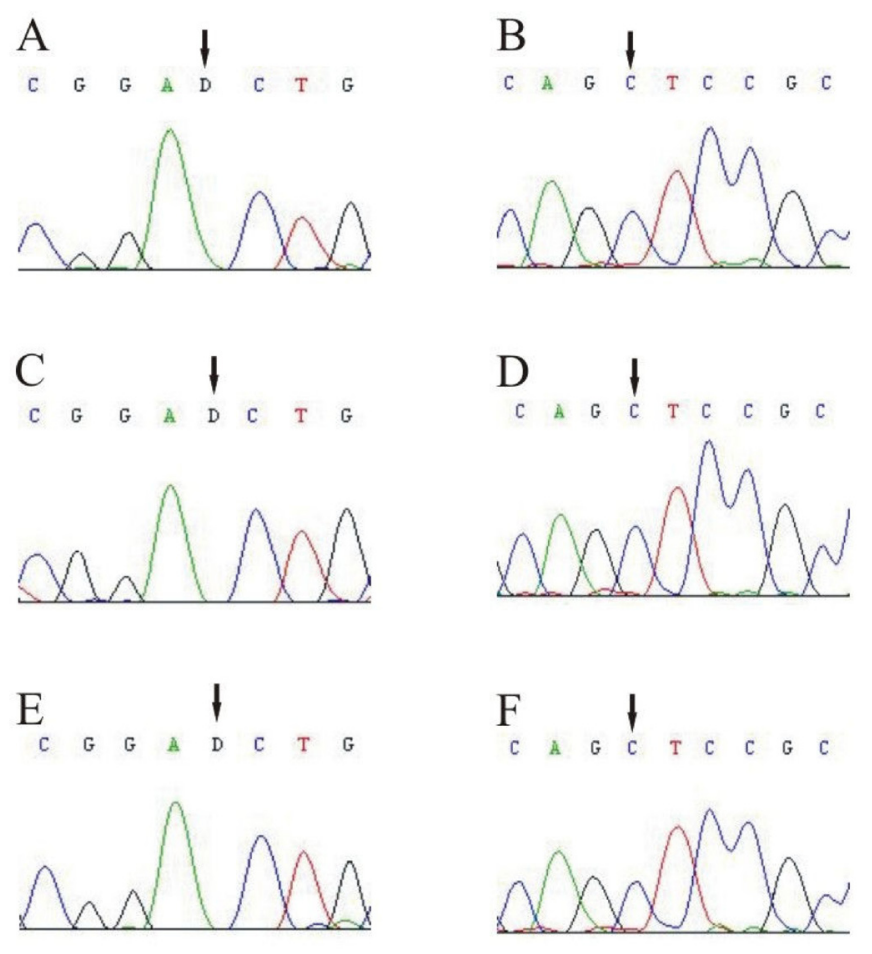

Figure 2. Chromatograms of the altered samples. A, C and E represent the sense strand sequencing; the arrows indicate the place of the deletion. B, D and F represent the antisense strand sequencing.

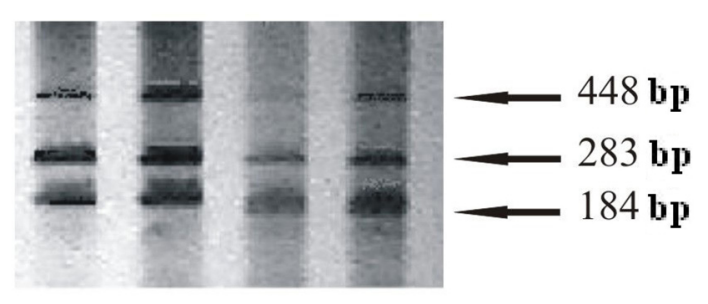

Figure 3. Digestion of the gene p15INK4b exon 2 by the $A l u \mathrm{I}$ enzyme (polyacrylamide gel); the fragment of 448 bp represents non-digestion due to the deletion of the base G in the DNA sense strand. The fragments of 184 and $283 \mathrm{bp}$ indicate the enzymatic digestion of the DNA antisense strand. 


\section{DISCUSSION}

Most authors suggest that more than $70 \%$ of all types of human cancers can be attributed to the genetics and epigenetic alterations of the tumor suppressor genes, mainly involving alterations in proteins associated with checkpoints in G1/S of the cell cycle (Wolff et al., 2005; Bartoletti et al., 2007).

Different mechanisms of inactivation of the INK4 genes have been observed, including homozygous deletions, mutations and hypermethylation of the promoter region. Homozygous deletions in the CDKN2A-CDKN2B (INK4 locus) region, which involves the p16 and p15 genes, are observed in 34 to $56 \%$ of glioblastomas and in a variety of other human cancers (Jen et al., 1994; Cairns et al., 1995; Moulton et al., 1995; Sherr, 1996; Schmidt et al., 1997; Simon et al., 1999).

In agreement with these and other studies, we found a homozygous deletion in exon 2 of the p15INK $4 \mathrm{~b}$ and exon 3 of the p16INK4a gene in two schwannoma samples. Although studies of these genes are less frequent in tumors of peripheral nerves, such as schwannomas, deletions are frequently observed in other tumors of the nervous system. Losses of the $9 p$ chromosome are often found in anaplastic meningioma and more rarely in atypical or benign meningiomas. Analyses of the $9 \mathrm{p} 21$ region indicate that more than $20 \%$ of the anaplastic meningiomas have a loss of heterozygosity of the p16 gene (Bostrom et al., 2001). Losses in the $9 \mathrm{p} 21$ region are also connected to the progression of oligodendrogliomas from grade II to grade III. In a series of tumors studied by Godfraind et al. (2003), deletions of 9p21 were observed in $62 \%$ of the oligodendrogliomas grade III.

Point mutations or small deletions of the $\mathrm{p} 15$ gene seem to be rare events. Yonghao et al. (1999) studied 357 samples of different primary tumors, and they observed that most of the deletions found involve genes $\mathrm{p} 15$ and $\mathrm{p} 16$ and that some tumors show loss of heterozygosity for all $9 \mathrm{p}$ region, but they did not observe small deletions or point mutations. When studying 35 cases of primary hepatocellular carcinoma with the PCR-SSCP technique, Qin et al. (1998) found a sample with the pattern of abnormal migration of a single strand in exon 2 of the p15 gene, but the sequencing revealed that it was a polymorphism of this exon, reinforcing the fact that small deletions and point mutations are rare events of inactivation of this gene in most tumors.

Although these events are uncommon, this study presented three samples with a guanine deletion at 63 codon (glu) only in the sense strand in the exon 2 of this gene, leading to a loss of heterozygosity of this gene, changing the product transcribed in this strand, and consequently, it can inactivate or modify the function of the protein produced, impairing the mechanism of the inhibitor action of CDKs. Point mutations, mainly in exon 2, promote the loss of inhibitory activity of p15 and p16 in relation to CDK4 (Reymond and Brent, 1995; Yoshida et al., 1995; Quelle et al., 1997).

Ghimenti et al. (2003) analyzed 61 samples of glioblastomas, in relation to the gene silencing of genes p14ARF and p16. They found homozygous deletions in $48 \%$ of the cases for the 16 gene and $54 \%$ for the p14ARF, and methylation of the promoter region in $13 \%$ of the cases for the p16 and $67 \%$ for the p14ARF. Methylation of the promoter region of the p14ARF gene is considered an initial event in the tumorigenesis of oligodendrogliomas (Watanabe et al., 2002), and point mutations restricted to exon $1 \beta$ are not frequently observed (Simon et al., 1999). The samples studied showed no alterations in the p14ARF; perhaps, epigenetic events may be responsible for the silencing of this gene. 
The inactivation of the tumor suppressor gene PTEN has been described in a variety of human tumors, including brain tumors (Ali et al., 1999). The loss of the PTEN function may occur by different mechanisms, including mutations, loss of heterozygosity of the PTEN locus on the 10q23 chromosome and epigenetic silencing of PTEN expression (Xiao et al., 2005; Ohgaki and Kleihues et al., 2007). Mutations in the PTEN gene occur frequently in gliomas, mainly in glioblastoma multiforme, indicating that alterations in the PTEN gene contribute to the neoplastic progression of gliomas (Xiao et al., 2005; Ohgaki, 2005; Ohgaki and Kleihues, 2007).

In our samples, we did not find any alteration in the PTEN gene. The PTEN gene is mutated mainly in malignant tumors, and our sample group consisted mainly of benign tumors. In relation to malignant tumors such as gliobastoma multiforme, the literature quotes a high frequency of alterations related to the PTEN gene. In a total of 634 cases of malignant glioma described in the literature, only 159 (24\%) showed mutations in the PTEN gene (Ali et al., 1999). Our group of patients had only 9 samples of glioblastomas and we found no alteration.

In summary, homozygous deletions of p15 and p16 were observed in two schwannomas and deletions of a single base occurred in three samples of p15, causing loss of heterozygosity of this gene. Homozygous deletions and loss of heterozygosity are often found in nervous system tumors, and they seem to be initial events in tumor formation. Although p14ARF share exon 2 of p16 through an alternative reading frame, changes in this gene were not observed in our samples; however, epigenetic events are silencing mechanisms that are very frequent in p14ARF, and analyses in this direction need to be carried out with our samples. We also did not find alterations in the PTEN, perhaps due to the fact that this gene is mainly related to the tumor progression process, being mainly mutated in gliobastomas, and that our group of glioblastoma patients was small.

\section{ACKNOWLEDGMENTS}

We are grateful to the patients who took part in this investigation. We thank Márcio Rogério Penha and Vanderci Massaro de Oliveira for technical help. Research supported by Conselho Nacional de Desenvolvimento Científico e Tecnológico (CNPq), Fundação de Amparo à Pesquisa do Estado de São Paulo (FAPESP) and Fundação de Apoio ao Ensino, Pesquisa e Assistência do Hospital das Clínicas da Faculdade de Medicina de Ribeirão Preto (FAEPA).

\section{REFERENCES}

Ali IU, Schriml LM and Dean M (1999). Mutational spectra of PTEN/MMAC1 gene: a tumor suppressor with lipid phosphatase activity. J. Natl. Cancer Inst. 91: 1922-1932.

Bartoletti R, Cai T, Nesi G, Roberta GL, et al. (2007). Loss of P16 expression and chromosome 9p21 LOH in predicting outcome of patients affected by superficial bladder cancer. J. Surg. Res. 143: 422-427.

Bostrom J, Meyer-Puttlitz B, Wolter M, Blaschke B, et al. (2001). Alterations of the tumor suppressor genes CDKN2A (p16(INK4a)), p14(ARF), CDKN2B (p15(INK4b)), and CDKN2C (p18(INK4c)) in atypical and anaplastic meningiomas. Am. J. Pathol. 159: 661-669.

Cairns P, Polascik TJ, Eby Y, Tokino K, et al. (1995). Frequency of homozygous deletion at p16/CDKN2 in primary human tumours. Nat. Genet. 11: 210-212.

Cánepa ET, Scassa ME, Ceruti JM, Marazita MC, et al. (2007). INK4 proteins, a family of mammalian CDK inhibitors with novel biological functions. IUBMB Life 59: 419-426.

Chu EC and Tarnawski AS (2004). PTEN regulatory functions in tumor suppression and cell biology. Med. Sci. Monit. 10: RA235-RA241.

Collins VP (2004). Brain tumours: classification and genes. J. Neurol. Neurosurg. Psychiatry 75 (Suppl. 2): ii2-ii11. 
Di Vizio D, Cito L, Boccia A, Chieffi P, et al. (2005). Loss of the tumor suppressor gene PTEN marks the transition from intratubular germ cell neoplasias (ITGCN) to invasive germ cell tumors. Oncogene 24: 1882-1894.

Ghimenti C, Fiano V, Chiado-Piat L, Chio A, et al. (2003). Deregulation of the p14ARF/Mdm2/p53 pathway and G1/S transition in two glioblastoma sets. J. Neurooncol. 61: 95-102.

Godfraind C, Rousseau E, Ruchoux MM, Scaravilli F, et al. (2003). Tumour necrosis and microvascular proliferation are associated with $9 \mathrm{p}$ deletion and CDKN2A alterations in 1p/19q-deleted oligodendrogliomas. Neuropathol. Appl. Neurobiol. 29: 462-471.

Gunther W, Skaftnesmo KO, Arnold H and Terzis AJ (2003). Molecular approaches to brain tumour invasion. Acta Neurochir. 145: 1029-1036.

Jen J, Harper JW, Bigner SH, Bigner DD, et al. (1994). Deletion of p16 and p15 genes in brain tumors. Cancer Res. 54: 6353-6358.

Kim WY and Sharpless NE (2006). The regulation of INK4/ARF in cancer and aging. Cell 127: 265-275.

Kukita Y, Tahira T, Sommer SS and Hayashi K (1997). SSCP analysis of long DNA fragments in low pH gel. Hum. Mutat. 10: 400-407.

Li DM and Sun H (1997). TEP1, encoded by a candidate tumor suppressor locus, is a novel protein tyrosine phosphatase regulated by transforming growth factor beta. Cancer Res. 57: 2124-2129.

Li J, Yen C, Liaw D, Podsypanina K, et al. (1997). PTEN, a putative protein tyrosine phosphatase gene mutated in human brain, breast, and prostate cancer. Science 275: 1943-1947.

Liu F, Wagner S, Campbell RB, Nickerson JA, et al. (2005). PTEN enters the nucleus by diffusion. J. Cell Biochem. 96 : 221-234.

Maehama T (2007). PTEN: its deregulation and tumorigenesis. Biol. Pharm. Bull. 30: 1624-1627.

Marino S, Krimpenfort P, Leung C, van der Korput HA, et al. (2002). PTEN is essential for cell migration but not for fate determination and tumourigenesis in the cerebellum. Development 129: 3513-3522.

Moulton T, Samara G, Chung WY, Yuan L, et al. (1995). MTS1/p16/CDKN2 lesions in primary glioblastoma multiforme. Am. J. Pathol. 146: 613-619.

Ohgaki K (2005). Genetic pathways to glioblastomas. Neuropathology 25: 1-7.

Ohgaki H and Kleihues P (2005). Epidemiology and etiology of gliomas. Acta Neuropathol. 109: 93-108.

Ohgaki H and Kleihues P (2007). Genetic pathways to primary and secondary glioblastoma. Am. J. Pathol. 170: $1445-1453$.

Ono Y, Tamiya T, Ichikawa T, Kunishio K, et al. (1996). Malignant astrocytomas with homozygous CDKN2/p16 gene deletions have higher Ki-67 proliferation indices. J. Neuropathol. Exp. Neurol. 55: 1026-1031.

Qin Y, Li B, Tan Y, Sun Z, et al. (1998). Mutation analysis of the p15 gene exon 2 in human primary hepatocarcinoma. Hua Xi Yi Ke Da Xue Xue Bao 29: 360-363.

Quelle DE, Cheng M, Ashmun RA and Sherr CJ (1997). Cancer-associated mutations at the INK4a locus cancel cell cycle arrest by p16INK4a but not by the alternative reading frame protein p19ARF. Proc. Natl. Acad. Sci. U. S. A. 94: 669-673.

Rasheed BK, McLendon RE, Friedman HS, Friedman AH, et al. (1995). Chromosome 10 deletion mapping in human gliomas: a common deletion region in 10q25. Oncogene 10: 2243-2246.

Rey JA, Bello MJ, Jimenez-Lara AM, Vaquero J, et al. (1992). Loss of heterozygosity for distal markers on 22q in human gliomas. Int. J. Cancer 51: 703-706.

Reymond A and Brent R (1995). p16 proteins from melanoma-prone families are deficient in binding to Cdk4. Oncogene 11: 1173-1178.

Rocha JCC (2001). Câncer do Sistema Nervoso Central. In: Genética Molecular do Câncer (Louro ID, Llerena JC Jr, Melo MSV, Ashton-Prolla P, et al., eds.). 2 ${ }^{\mathrm{a}}$ ed. MSG Produção Editorial, São Paulo, 243-251.

Sansal I and Sellers WR (2004). The biology and clinical relevance of the PTEN tumor suppressor pathway. J. Clin. Oncol. 22: 2954-2963.

Schmidt EE, Ichimura K, Messerle KR, Goike HM, et al. (1997). Infrequent methylation of CDKN2A(MTS1/p16) and rare mutation of both CDKN2A and CDKN2B(MTS2/p15) in primary astrocytic tumours. Br. J. Cancer 75: 2-8.

Sherr CJ (1996). Cancer cell cycles. Science 274: 1672-1677.

Sherr CJ and Roberts JM (1999). CDK inhibitors: positive and negative regulators of G1-phase progression. Genes Dev. 13: 1501-1512.

Simon M, Koster G, Menon AG and Schramm J (1999). Functional evidence for a role of combined CDKN2A (p16p14(ARF))/CDKN2B (p15) gene inactivation in malignant gliomas. Acta Neuropathol. 98: 444-452.

Watanabe T, Nakamura M, Kros JM, Burkhard C, et al. (2002). Phenotype versus genotype correlation in oligodendrogliomas and low-grade diffuse astrocytomas. Acta Neuropathol. 103: 267-275. 
Wolff EM, Liang G and Jones PA (2005). Mechanisms of disease: genetic and epigenetic alterations that drive bladder cancer. Nat. Clin. Pract. Urol. 2: 502-510.

Xiao A, Yin C, Yang C, Di Cristofano A, et al. (2005). Somatic induction of Pten loss in a preclinical astrocytoma model reveals major roles in disease progression and avenues for target discovery and validation. Cancer Res. 65 : 5172-5180.

Yonghao T, Qian H, Chuanyuan L and Yandell DW (1999). Deletions and point mutations of p16,p15 gene in primary tumors and tumor cell lines. Chin. Med. Sci. J. 14: 200-205.

Yoshida S, Todoroki T, Ichikawa Y, Hanai S, et al. (1995). Mutations of p16Ink4/CDKN2 and p15Ink4B/MTS2 genes in biliary tract cancers. Cancer Res. 55: 2756-2760. 